\title{
Search for ttH in Run 1 at the LHC
}

\author{
Christopher Neu* \\ University of Virginia \\ E-mail: chris.neu@virginia.edu
}

\begin{abstract}
With the discovery in 2012 of a Higgs boson at the LHC, the focus of the Higgs physics campaigns at the ATLAS and CMS experiments has transitioned from the search for the Higgs to the pursuit of the complete characterization of this new particle. This is an essential step that is necessary in the determination of whether this is the Higgs boson of the standard model, or a component of some other, more exotic explanation of the fundamental world. Principal among these characterizations is the understanding of the interaction strength, or coupling, between this Higgs boson and the other known fundamental particles; among these couplings, the largest and arguably the most important is that between the Higgs and the top quark, the most massive of the known fundamental particles. The only direct probe of this coupling is through the observation of Higgs boson production in association with top quarks at the LHC. In this talk, the search strategy and current status will be described, focusing on the results from the 2010-2012 Run 1 of the LHC, with also an eye toward future studies in Run 2. The observation of this process is one of the highest-priority aspects of the current LHC physics program.
\end{abstract}

Frontiers of Fundamental Physics 14 - FFP14,

15-18 July 2014

Aix Marseille University (AMU) Saint-Charles Campus, Marseille

${ }^{*}$ Speaker. 